\title{
COERCIVE ESTIMATES AND EXISTENCE OF SOLUTIONS FOR A MODEL OF ONE-DIMENSIONAL VISCOELASTICITY WITH A NON-INTEGRABLE MEMORY FUNCTION
}

\author{
MICHAEL RENARDY
}

\begin{abstract}
We consider a model equation for one-dimensional motions of viscoelastic materials with a non-integrable memory function. We prove existence of solutions to the initial value problem globally in time when the data are smooth and small, and locally in time when the data are smooth but large. The proof of existence is based on coercive estimates for the linearized problem. Such estimates exploit the singular nature of the memory function.
\end{abstract}

1. Introduction. We consider integrodifferential equations of the form:

$$
\begin{gathered}
u_{t t}(x, t)=g\left(u_{x}(x, t)\right)_{x}+\int_{-\infty}^{t} m(t-\tau) h\left(u_{x}(x, t), u_{x}(x, \tau)\right)_{x} d \tau \\
+f(x, t), x \in[0,1], t>0
\end{gathered}
$$

under the following assumptions:

(i) The functions $g$ and $h$ are of class $C^{4}$ and $C^{5}$, respectively.

(ii) The function $\operatorname{tm}(t)$ is integrable on $(0, \infty)$.

(iii) $g^{\prime}(0)>0$, and $h(p, p)=0, h_{, 1}(p, p)=-h_{, 2}(p, p)>0$ for every $p \in \mathbf{R}$. Here $h_{, i}$ denotes the derivative with respect to the $i$ th argument.

(iv) $m \in W^{1,1}\left[t_{0}, \infty\right)$ for every $t_{0}>0$ and $m>0, m^{\prime}<0$.

Equations of the form (1) can be used to model one-dimensional motions of viscoelastic materials in both shear and elongation (see, e.g., [6, Chapter I]). The kernel $m$ is called the memory function. The assumptions (i)-(iv) are physically natural ones. The variable $u$ represents the displacement. For simplicity, we shall confine our attention to homogeneous Dirichlet boundary conditions

$$
\begin{aligned}
& u(0, t)=u(1, t)=0, t>0 \\
& \text { Copyright }(1988 \text { Rocky Mountain Mathematics Consortium }
\end{aligned}
$$


the results that follow can easily be modified to accommodate more general boundary conditions. In addition, we prescribe the initial history for $t \leq 0$ :

$$
u(x, t)=u_{0}(x, t), x \in[0,1], t \leq 0 .
$$

For the case where $m$ is smooth,problem (1)-(3) or special cases thereof have been considered by a number of authors; see Chapters III and IV of [6] for a summary of results and references to the literature. It is known that solutions exist locally in time if the data $f$ and $u_{0}$ are sufficiently smooth and globally in time if, in addition, it is assumed that the data are small. The proofs rely heavily on treating (1) as a perturbation of a hyperbolic equation. This can no longer be done if $m$ has a singularity at the origin.

In [2], [3] a special case of (1) is considered under assumptions which allow a singularity in $m$. The proofs in [2], [3] proceed by considering a sequence of approximating problems for which $m$ is regular and establishing uniform energy estimates which allow passage to the limit. On the other hand, it is known that for a wide class of memory functions with nonintegrable singularities at the origin there are coercive estimates for the linearization of (1) similar to those for parabolic equations (see [1] , [5]). This suggests that the energy method may not be the optimal basis of an existence theory. The goal of this paper is to develop an existence theory which exploits the parabolic character of the equation. We make the following assumption on $m$ :

(v) Let

$$
G(t):=\int_{t}^{\infty} m(s) d s
$$

and let $\hat{G}$ denote the Fourier transform of $G$ :

$$
\hat{G}(\omega):=\int_{0}^{\infty} G(t) e^{-i \omega t} d t .
$$

Then there is a constant $C>0$ such that, for every $\omega \in \mathbf{R}$, we have $|\Re \hat{G}(\omega)| \geq C|\Im \hat{G}(\omega)|$.

We note that assumption (ii) implies that $G$ is integrable. Assumption (v) implies that $m$ cannot be integrable at the origin. Assumption 
(v) is satisfied if, for example, $G(t)$ behaves like an inverse power of $t$ near $t=0$.

In the following, we shall assume that the history $u_{0}$ satisfies the boundary conditions (2) and the differential equation (1) for $t<0$. Of course we can always make the history satisfy the differential equation by defining $f(x, t)$ appropriately for $t<0$. It should be kept in mind, however, that smoothness assumptions on $f$ at $t=0$ actually represent compatibility conditions between the prescribed history and the evolution for positive $t$. Henceforth we shall adopt the point of view that we seek a solution of equation (1) and boundary condition (2) for all $t$, but that the solution for $t<0$ is actually known.

2. Existence of solutions for small data. In this section we consider the case where $f$ and $u_{0}$ are small in appropriate norms. Since it will turn out that in this case $u_{0}$ is uniquely determined by $f$, we shall for the moment ignore the initial condition (3) and seek a solution of (1) and (2) for given $f$. The existence proof will be based on the implicit function theorem and the properties of the linearized equation. Before we state a precise theorem, we must first define some function spaces.

Definition. Let $X$ be a Hilbert space. $B y H_{G}^{k}(\mathbf{R} ; X)$ we denote the set of all functions $f: \mathbf{R} \rightarrow X$ such that the Fourier transform of $f$ has the form $\hat{f}(\omega)=\hat{G}(\omega) \hat{g}(\omega)$ where $g$ lies in $H^{k}(\mathbf{R} ; X)$.

Since $G$ is positive and monotone decreasing, $\hat{G}(\omega)$ does not vanish; moreover, $\hat{G}(\omega)$ tends to zero and $|\omega \hat{G}(\omega)|$ tends to infinity as $|\omega| \rightarrow \infty$. Hence it follows that $H^{k+1}(\mathbf{R} ; X) \subset H_{G}^{k}(\mathbf{R} ; X) \subset H^{k}(\mathbf{R} ; X)$. We shall prove the following result:

THEOREM. Let $f \in H_{G}^{1}\left(\mathbf{R} ; L^{2}(0,1)\right)$ be given and assume that the norm of $f$ is sufficiently small. Then there exists a solution $u$ of $(1)$ and (2) for all $t \in \mathbf{R}$ and we have $u \in H_{G}^{3}\left(\mathbf{R} ; L^{2}(0,1)\right) \cap H^{2}\left(\mathbf{R} ; H^{2}(0,1)\right)$. The solution $u$ is unique within the class of functions which have small norm in this space.

The proof will be based on the implicit function theorem. We define 
the map $F$ by

(6)

$F(u)(x, t)=u_{t t}(x, t)-g\left(u_{x}(x, t)\right)_{x}-\int_{-\infty}^{t} m(t-\tau) h\left(u_{x}(x, t), u_{x}(x, \tau)\right)_{x} d \tau$.

The theorem follows if we can show the following two statements:

1. $F$ is a continuously differentiable map from $Z:=H_{G}^{3}\left(\mathbf{R} ; L^{2}(0,1)\right) \cap$ $H^{2}\left(\mathbf{R} ; H^{2}(0,1) \cap H_{0}^{1}(0,1)\right)$ into $H_{G}^{1}\left(\mathbf{R} ; L^{2}(0,1)\right)$.

2. The Fréchet derivative $D F(0)$ is invertible.

In verifying statement 1 , the first two terms in (6) present no difficulty (the term $g\left(u_{x}\right)_{x}$ lies in $\left.H^{2}\left(\mathbf{R} ; L^{2}(0,1)\right) \subset H_{G}^{1}\left(\mathbf{R} ; L^{2}(0,1)\right)\right)$. We shall now focus on the integral term. After integration by parts, this term reads as follows:

$$
\begin{aligned}
& \frac{\partial}{\partial x} \int_{-\infty}^{t} G(t-\tau)_{h, 2}\left(u_{x}(x, t), u_{x}(x, \tau)\right) u_{x t}(x, \tau) d \tau \\
= & \frac{\partial}{\partial x} \int_{0}^{\infty} G(s) h,_{2}\left(u_{x}(x, t), u_{x}(x, t-s)\right) u_{x t}(x, t-s) d s .
\end{aligned}
$$

We first note that the map $H$ given by

$$
H(u)(x, t)=\int_{0}^{\infty} G(s) h,_{2}\left(u_{x}(x, t), u_{x}(x, t-s)\right) u_{x t}(x, t-s) d s
$$

is continuously differentiable from $Z$ into $H^{1}\left(\mathbf{R} ; H^{1}(0,1)\right)$. To see this, we simply have to observe that $G$ is integrable and that the maps $H_{s}$ defined by

$$
H_{s}(u)(x, t)=h,,_{2}\left(u_{x}(x, t), u_{x}(x, t-s)\right) u_{x t}(x, t-s)
$$

are continuously differentiable from $Z$ into $H^{1}\left(\mathbf{R} ; H^{1}(0,1)\right)$, uniformly with respect to $s$. In order to complete the proof of statement 1 , we have to show that $H$ actually is continuously differentiable from $Z$ into the smaller space $H_{G}^{1}\left(\mathbf{R} ; H^{1}(0,1)\right)$. For this, let us consider the time derivative of $H(u)$ :

$$
\begin{aligned}
& \frac{\partial}{\partial t} H(u)(x, t)=\int_{0}^{\infty} G(s)\left[h,_{12}\left(u_{x}(x, t), u_{x}(x, t-s)\right) u_{x t}(x, t)\right. \\
& \left.\quad+h, 22\left(u_{x}(x, t), u_{x}(x, t-s)\right) u_{x t}(x, t-s)\right] u_{x t}(x, t-s) d s \\
& \quad+\int_{0}^{\infty} G(s) h,_{2}\left(u_{x}(x, t), u_{x}(x, t-s)\right) u_{x t t}(x, t-s) d s
\end{aligned}
$$


The first term on the right hand side of (10) takes values in $H^{1}(\mathbf{R}$; $\left.H^{1}(0,1)\right) \subset H_{G}^{0}\left(\mathbf{R} ; H^{1}(0,1)\right)$ and presents no problem. The second term can be split as follows:

$$
\begin{aligned}
& \int_{0}^{\infty} G(s) h,_{2}\left(u_{x}(x, t-s) u_{x}(x, t-s)\right) u_{x t t}(x, t-s) d s \\
& +\int_{0}^{\infty} G(s)\left[h, 2\left(u_{x}(x, t), u_{x}(x, t-s)\right)\right. \\
& \left.\quad-h,,_{2}\left(u_{x}(x, t-s), u_{x}(x, t-s)\right)\right] u_{x t t}(x, t-s) d s .
\end{aligned}
$$

The first term in (11) is in convolution form and it is clear that it represents a continuously differentiable map from $Z$ into $H_{G}^{0}\left(\mathbf{R} ; H^{1}(0,1)\right)$. In the second term, we integrate by parts with respect to $s$ and obtain (12)

$$
\begin{aligned}
& -\int_{0}^{\infty} G(s)\left[h,,_{22}\left(u_{x}(x, t), u_{x}(x, t-s)\right)-h,,_{22}\left(u_{x}(x, t-s), u_{x}(x, t-s)\right)\right. \\
& \left.-h_{, 12}\left(u_{x}(x, t-s), u_{x}(x, t-s)\right)\right] u_{x t}^{2}(x, t-s) d s \\
& -\int_{0}^{\infty} m(s)\left[h, 2\left(u_{x}(x, t), u_{x}(x, t-s)\right)-h,_{2}\left(u_{x}(x, t-s), u_{x}(x, t-s)\right)\right] \\
& \quad \cdot u_{x t}(x, t-s) d s .
\end{aligned}
$$

The first term in (12) represents a continuously differentiable map with values in $H^{1}\left(\mathbf{R} ; H^{1}(0,1)\right)$. In the second term, we make the substitution

$$
\begin{gathered}
h_{2}\left(u_{x}(x, t), u_{x}(x, t-s)\right)-h,_{2}\left(u_{x}(x, t-s), u_{x}(x, t-s)\right) \\
=s \int_{0}^{1} h,_{12}\left(u_{x}(x, t-\alpha s), u_{x}(x, t-s)\right) u_{x t}(x, t-\alpha s) d \alpha
\end{gathered}
$$

Since $s m(s)$ is integrable, it is clear that after substituting (13) into (12) we obtain an expression which also represents a continuously differentiable map with values in $H^{1}\left(\mathbf{R} ; H^{1}(0,1)\right)$. This concludes the verification of statement 1 .

To verify statement 2 , we expand $u$ in a sine series,

$$
u(x, t)=\sum_{k=1}^{\infty} u_{k}(t) \sin k \pi x
$$


and we denote by $\hat{u}_{k}(\omega)$ the Fourier transform of $u_{k}$. Analogous notation is used for $f$. The statement that $f \in H_{G}^{1}\left(\mathbf{R} ; L^{2}(0,1)\right)$ translates into

$$
\sum_{k=1}^{\infty} \int_{-\infty}^{\infty}\left|\hat{f}_{k}(\omega)\right|^{2} \frac{1+|\omega|^{2}}{|\hat{G}(\omega)|^{2}} d \omega<\infty
$$

and the statement that $u \in Z$ translates into

$$
\sum_{k=1}^{\infty} \int_{-\infty}^{\infty}\left|\hat{u}_{k}(\omega)\right|^{2}\left[\frac{\omega^{6}+1}{|\hat{G}(\omega)|^{2}}+k^{4}\left(w^{4}+1\right)\right] d \omega<\infty
$$

The Fréchet derivative $D F(0)$ is the map

$$
\begin{aligned}
D F(0) u(x, t)= & u_{t t}(x, t)-g^{\prime}(0) u_{x x}(x, t) \\
& +\int_{-\infty}^{t} G(t-\tau) h,_{2}(0,0) u_{x x t}(x, \tau) d \tau
\end{aligned}
$$

The equation $D F(0) u=f$ transforms into

$$
\left(-\omega^{2}+g^{\prime}(0) k^{2} \pi^{2}-h, 2(0,0) \hat{G}(\omega) i \omega k^{2} \pi^{2}\right) \hat{u}_{k}(\omega)=\hat{f}_{k}(\omega) .
$$

Let us denote the term multiplying $\hat{u}_{k}(\omega)$ on the left hand side by $N(k, \omega)$. Since $G$ is positive, monotone decreasing and convex, the real part of $\hat{G}(\omega)$ is always strictly positive [4], and hence $N(k, \omega)$ is never zero. We also have $|N(k, \omega)| \geq|\Im N(k, \omega)|$, and because of assumption (v) the latter is bounded from below by a constant times $k^{2}|\omega \hat{G}(\omega)|$. Hence (18) implies an estimate of the form

$$
\left|\hat{u}_{k}(\omega)\right| \leq C \frac{\left|\hat{f}_{k}(\omega)\right|}{k^{2}(1+|\omega \hat{G}(\omega)|)} .
$$

Using this, we can bound the second and third term on the left of (18) in terms of the right hand side, and hence we can also bound the first term. One easily concludes that the norm of $u$ defined by (16) can be bounded in terms of the norm of $f$ defined by (15).

REMARK. We still need to discuss the meaning of this result in terms of the original initial-history value problem (1)-(3). It is clear that for 
any sufficiently well-behaved history $u_{0}$ we can define $f$ for $t<0$ by using equation (1) (with $u_{0}$ substituted for $u$ ). We can then use the above theorem to find a solution $u$ for all $t$, but it takes a proof that we actually recover $u=u_{0}$ for $t<0$. This follows if the solution operator for (1), (2) (posed for all $t \in \mathbf{R}$ ) is causal, i.e., if the solution $u$ for $t<0$ depends only on the values of $f$ for $t<0$. In this case we can argue as follows: Take a well-behaved extension of $u_{0}$ for positive time (let us call it $\tilde{u}$ ) and define a corresponding forcing function $\tilde{f}$ by using equation (1). If the solution operator is causal, then $f=\tilde{f}$ for $t<0$ implies that $u=\tilde{u}=u_{0}$ for $t<0$. Note now that the assumptions on $G$ and on the signs of $g^{\prime}(0)$ and $h, 2(0,0)$ permit the above estimates for $N(k, \omega)$ to be extended for $\omega$ in the lower half plane. It follows from this that $D F(0)^{-1}$ is causal. The causality of the solution operator now follows from the iteration which underlies the proof of the implicit function theorem.

3. Local existence of solutions for large data. In this section, we shall prove existence of solutions without a smallness assumption on $u_{0}$ and $f$. In this case, however, we shall only obtain existence of solutions for short time. Before we state precise assumptions and a theorem, we shall first outline the basic procedure. Let $F(u)$ be defined as in (6); after an integration by parts we obtain

$$
\begin{aligned}
F(u)(x, t)= & u_{t t}(x, t)-g\left(u_{x}(x, t)\right)_{x} \\
& +\int_{0}^{\infty} G(s)\left[h,_{2}\left(u_{x}(x, t), u_{x}(x, t-s)\right) u_{x t}(x, t-s)\right]_{x} d s .
\end{aligned}
$$

Let $\tilde{u}$ be a smooth extension of $u_{0}$ for positive time and let $\tilde{f}=F(\tilde{u})$. Moreover, let $v=u-\tilde{u}$ and $\hat{f}=f-\tilde{f}$. That is, we have $\hat{f}(x, t)=0$ for $t<0$ and we are looking for solutions which satisfy $v(x, t)=0$ for $t<0$. The equation of motion now assumes the form 
(21)

$$
\begin{aligned}
& H(v)(x, t):=v_{t t}(x, t)+\frac{\partial}{\partial x}\left[\left(-g\left(\tilde{u}_{x}(x, t)+v_{x}(x, t)\right)+g\left(\tilde{u}_{x}(x, t)\right)\right)\right. \\
& +\int_{0}^{\infty} G(s)\left(h,_{2}\left(\tilde{u}_{x}(x, t)+v_{x}(x, t), \tilde{u}_{x}(x, t-s)\right.\right. \\
& \left.+v_{x}(x, t-s)\right) v_{x t}(x, t-s) \\
& +\left[h, 2\left(\tilde{u}_{x}(x, t)+v_{x}(x, t), \tilde{u}_{x}(x, t-s)+v_{x}(x, t-s)\right)\right. \\
& \left.\left.\left.-h, 2\left(\tilde{u}_{x}(x, t), \tilde{u}_{x}(x, t-s)\right)\right] \tilde{u}_{x t}(x, t-s)\right) d s\right] \\
& =\hat{f}(x, t) .
\end{aligned}
$$

As in the previous section, we shall use an implicit function argument. We therefore have to consider the linearization of equation (21). We have

$$
\begin{aligned}
& D H(0) v(x, t)=v_{t t}(x, t)+\frac{\partial}{\partial x}\left[-g^{\prime}\left(\tilde{u}_{x}(x, t)\right) v_{x}(x, t)\right. \\
& \quad+\int_{0}^{\infty} G(s) h,_{2}\left(\tilde{u}_{x}(x, t), \tilde{u}_{x}(x, t-s)\right) v_{x t}(x, t-s) d s \\
& \quad+\int_{0}^{\infty} G(s)\left(h,,_{12}\left(\tilde{u}_{x}(x, t), \tilde{u}_{x}(x, t-s)\right) v_{x}(x, t)\right. \\
& \left.\left.\quad+h, 22\left(\tilde{u}_{x}(x, t), \tilde{u}_{x}(x, t-s)\right) v_{x}(x, t-s)\right) \tilde{u}_{x t}(x, t-s) d s\right] .
\end{aligned}
$$

Let $Z=H_{G}^{3}\left(\mathbf{R} ; L^{2}(0,1)\right) \cap H^{2}\left(\mathbf{R} ; H^{2}(0,1) \cap H_{0}^{1}(0,1)\right), Y=H_{G}^{1}(\mathbf{R}$; $\left.L^{2}(0,1)\right)$ and let $T>0$ be given. By $Z_{T}, Y_{T}$ we denote the set of all functions defined on $(-\infty, T)$ which have an extension to all of $\mathbf{R}$ which lies in $Z$, or respectively, $Y$. By $Z_{T, 0}$ and $Y_{T, 0}$ we denote the space of all functions in $Z_{T}$, or, respectively, $Y_{T}$ which vanish on $(-\infty, 0)$. The norm in $Z_{T}$ is defined as the infimum of the norms of all extensions which lie in $Z$. With these definitions we can now state our existence result.

THEOREM. Let $\tilde{u} \in Z_{T}$ and $\hat{f} \in Y_{T, 0}$ be given. Then there exists $T^{\prime} \in(0, T]$ such that $(21)$ has a uniquely determined solution $v \in Z_{T^{\prime}, 0}$.

For the proof, we first note that it follows from the arguments given in the previous section that $H$ is a continuously differentiable map from 
$Z_{T, 0}$ into $Y_{T, 0}$. The theorem now follows from the implicit function theorem and the following two assertions:

1. The norm of $\hat{f}$ in $Y_{T^{\prime}, 0}$ tends to zero as $T^{\prime} \rightarrow 0$.

2. If $T^{\prime}$ is chosen small enough, then $D H(0)$ (as a map from $Z_{T^{\prime}, 0}$ to $\left.Y_{T^{\prime}, 0}\right)$ is invertible.

Assertion 1 is clearly true if $\hat{f}$ is of class $C^{\infty}$ and the general statement follows from the fact that such functions are dense in $Y_{T, 0}$. We now make the following observations:

a. If $v \in H^{k+1}\left(\left(-\infty, T^{\prime}\right] ; X\right)$ and $v=0$ for $t<0$, then the norm of $v$ in $H^{k}\left(\left(-\infty, T^{\prime}\right] ; X\right)$ has a bound of order $T^{\prime}$ times the norm in $H^{k+1}\left(\left(-\infty, T^{\prime}\right] ; X\right)$.

b. If $v \in H^{k+1}\left(\left(-\infty, T^{\prime}\right] ; X\right)$ and $v=0$ for $t<0$, then $v$ can be extended to a function in $H^{k+1}(\mathbf{R} ; X)$; let us again denote the extended function by $v$. The extension can be done in such a way that the norm of $v$ in $H^{k}(\mathbf{R} ; X)$ is still of order $T^{\prime}$. Moreover, for every $\varepsilon>0$ there is an estimate of the form

$$
\|v\|_{H_{G}^{k}(\mathbf{R} ; X)} \leq \varepsilon\|v\|_{H^{k+1}(\mathbf{R} ; X)}+C^{\prime}(\varepsilon)\|v\|_{H^{k}(\mathbf{R} ; X)} .
$$

This can be shown by taking Fourier transforms and observing that $|\omega \hat{G}(\omega)| \rightarrow \infty$ as $|\omega| \rightarrow \infty$. Hence, if $v \in H^{k+1}\left(\left(-\infty, T^{\prime}\right] ; X\right)$ and $T^{\prime}$ is small, then the norm of the extension in $H_{G}^{k}(\mathbf{R} ; X)$ is small.

By using these observations and doing a term by term analysis of $D H(0)$ similar to the discussion of $F$ in $\S 2$ we can show that for small $T^{\prime}$ the operator $D H(0)$ from $Z_{T^{\prime}, 0}$ to $Y_{T^{\prime}, 0}$ is a small perturbation of the operator $Q$ defined by

$$
Q v(x, t)=v_{t t}(x, t)+\frac{\partial}{\partial x} \int_{0}^{\infty} G(s) h,_{2}\left(\tilde{u}_{x}(x, 0), \tilde{u}_{x}(x, 0)\right) v_{x t}(x, t-s) d s .
$$

Let us define a new operator $\tilde{Q}$ by adding to $Q$ the perturbation

$$
\frac{\partial}{\partial x}\left(h, 2\left(\tilde{u}_{x}(x, 0), \tilde{u}_{x}(x, 0)\right) v_{x}(x, t)\right) .
$$

This perturbation is small if $T^{\prime}$ is small. The operator $\tilde{Q}$ can now be analyzed in a fashion similar to the analysis of $D F(0)$ in $\S 2$; we only 
have to replace the sine series by an expansion in eigenfunctions of the operator

$$
\frac{\partial}{\partial x}\left(h,_{2}\left(\tilde{u}_{x}(x, 0), \tilde{u}_{x}(x, 0)\right) \frac{\partial}{\partial x}\right) .
$$

Hence the operator $\tilde{Q}$ has an inverse from $Y$ to $Z$. As before, we can show that this inverse is a causal operator and hence it also maps $Y_{T^{\prime}, 0}$ to $Z_{T^{\prime}, 0}$. Since $\tilde{Q}$ is a small perturbation of $D H(0)$ for small $T^{\prime}$, we obtain the invertibility of $D H(0)$. This completes the proof.

Acknowledgement. This research was supported by the National Science Foundation under Grant DMS-8796241.

\section{REFERENCES}

1. R.C. Grimmer and A.J. Pritchard, Analytic resolvent operators for integral equations in Banach spaces, J. Diff. Eq. 50 (1983), 234-259.

2. W.J. Hrusa and M. Renardy, On a class of quasilinear partial integrodifferential equations with singular kernels, J. Diff. Eq. 64 (1986), 195-220.

3. $\longrightarrow$ and A model equation for viscoelasticity with a strongly singular kernel, SIAM J. Math. Anal. 19 (1988).

4. J.A. Nohel and D.F. Shea, Frequency domain methods for Volterra equations, Adv. Math. 22 (1976), 278-304.

5. G. Da Prato, M. Ianelli and E. Sinestrari, Regularity of solutions of a class of linear integrodifferential equations in Banach spaces, J. Integral Eq. 8 (1985), 27-40.

6. M. Renardy, W.J. Hrusa and J.A. Nohel, Mathematical Problems in Viscoelasticity, Longman 1987.

Department of Mathematics, Virginia Polytech State Univerísity, BLACKSBURG, VA 24061 